\title{
Early Outline Evaluation of Genotyping Costs of Pharmacogenomics
}

\section{Angela De Monaco', Massimiliano Berretta ${ }^{2}$, Sergio Pugliese ${ }^{3}$, Daniela Valente ${ }^{4}$ and Raffaele Di Francia ${ }^{4 *}$}

${ }^{1}$ Laboratory of Molecular Haematology, National Cancer Institute, Fondazione "G. Pascale" IRCCS, Naples, Italy

${ }^{2}$ Department of medical Oncology, CRO National Cancer Institute, IRCCS Aviano (PN), Italy

${ }^{3}$ CETAC Research Center, Caserta, Italy

${ }^{4}$ Italian Association of Pharmacogenomics and Molecular Diagnostics, Naples, Italy

Keywords: Pharmacogenomics; Genotyping methods; Molecular diagnostics; Pharmacoeconomy; Clinical laboratory

Abbreviations: PG: Pharmacogenetics and pharmacogenomics; NICE: National Institute for Health and Clinical Excellence; SNPs: Single Nucleotide Polymorphisms; SSCP: Single-Strand Conformational Polymorphism; FRET: Fluorescent Resonance Energy Transfer; MALDI TOF: Matrix-Assisted Laser Desorption/Ionization Time of Flight; ASA-PCR: Allele Specific Amplification-Polymerase Chain Reaction; RFLP: Restriction Fragment Length Polymorphism; D-HPLC: Denaturing-High Performance Liquid Chromatography; FDA: Food and Drug administration; CE: European Community; UGT1A1: Uridinediphosphate Glucuronosyl Transferase 1A1

\section{Introduction}

Promise in the future, a disease could be ranked into genetic categories, allowing bespoke tailoring of medicine to maximize therapeutic effects and to reduce the potential for adverse drug response.

As genomics-based technologies are widely introduced in clinical laboratories testing setting, the risks of mishandling or misinterpreting data from patient's sample analyses becomes a significant consideration with especially dramatic consequences where the test becomes commercially available to the public [1].

Generally, genotyping is performed either by custom service laboratories or academic referenced laboratories, as well as by using commercial kits (when available). In the USA, diagnostics products are regulated by the Food and Drug Administration (FDA), whereas diagnostic services are under the rules of the Clinical Laboratory Improvement Act (CLIA). In Europe this field is covered by in vitro Diagnostic (IVD) directive, without a distinction between commercial products (used by laboratories) and diagnostics service. In both case, a voluntary list of international laboratories (with CLIA certification in the US) able to perform genetic tests can be found on the National Institute of Health-funded website named GeneTests ${ }^{\mathrm{TM}}$ www.ncbi.nlm.nih.gov/sites/GeneTests/lab?db=GeneTests, although only a small minority of genetic tests listed on this site are Pharmacogenomics (PG) tests. Clinical laboratories may develop and validate tests in-house ("home-brew") and perform them as a laboratory service; which may further reduce the cost of analysis [2].

Pharmaceutical and Biotech companies frequently develop their own clinical pharmacokinetic and pharmacodynamic tests for new drug studies. They are required to have validated assays for human clinical phase III trials complying with current Good Clinical Practice guidelines for FDA or EMEA submission purposes. This involves testing patients as potential recipients before the administration of the drug. This difference poses an ethical dilemma for pharmaceutical companies, especially if inadequate testing excludes some patients who might benefit from receiving the drug or, conversely, long-term dosing continues with a treatment that does not have good clinical efficacy. Pharmaceutical companies should be involved with the initial development of PG assays because they have the primary data and information necessary for this stage of assay development. However, this assay development activity should be transferred to outside referenced laboratories, clinical core laboratories in academic health centers, or established Clinical Research Organizations when research and development transit into clinical application because these independent external sites are able to handle this function [3].

Reimbursement or payment for genetic testing is another topic of considerable consequence that is already creating controversy among health maintenance organizations, healthcare providers, and the patients themselves. One can predict, however, that health insurance companies will be very interested in patient PG testing to document the proper dosing of expensive prescription drugs and hence reduce the incidence or risks of adverse drug reactions. It will be interesting to see whether insurers will consider PG testing to be a cost-effective alternative to the current trial-and-error approach to dosage regulation. However, if the detection of these genetic variants is routinely incorporated either into clinical practice or large clinical trials, knowledge concerning the predictive value of $P G$ which will eventually enable the individualization of optimized therapy could be gained [4].

Several methods to assess the quality of cost-effectiveness, costutility and cost-benefit of PG tests have become available. A relevant example is the National Institute for Health and Clinical Excellence (NICE). NICE forms a Diagnostic Advisory committee, which stimulates Pharma and Academic communities to produce a robust set of data, including the design and data source, for economic models of healthcare [5].

Relative costs of PG tests, here, were evaluated by "manually cured criteria" due to lack of specific guidelines.

We wish to stress that the cost-effectiveness, cost-utility and costbenefit analysis (i.e. toxicity-related hospitalization) are not considered here.

The goal of this issue is to provide information on the advantages and limitations, in terms of costs, of the most common available methods for molecular detection of polymorphisms related to drug therapy.

*Corresponding author: Raffaele Di Francia, Hematology and stem cell transplantation, National Cancer Institute, Fondazione 'G.Pascale', IRCCS, via Mariano Semmola, 80131 Naples, Italy, Tel: +39 0815903636; Fax; +39 0815903833 ; E-mail: raffaele.difrancia@istitutotumori.na.it

Received September 26, 2013; Accepted January 31, 2014; Published February 07, 2014

Citation: Monaco AD, Berretta M, Pugliese S, Valente D, Francia RD (2014) Early Outline Evaluation of Genotyping Costs of Pharmacogenomics. J Pharmacogenomics Pharmacoproteomics 5: 123. doi:10.4172/2153-0645.1000123

Copyright: (c) 2014 Monaco AD, et al. This is an open-access article distributed under the terms of the Creative Commons Attribution License, which permits unrestricted use, distribution, and reproduction in any medium, provided the original author and source are credited. 
We believe that retrospective and prospective trials evaluating the pharmacoeconomic impact of genotyping testing will provide answers on the possibility to incorporate PG testing into routine clinical practice.

\section{Current Genotyping Methods for Known Mutations}

The most popular platforms for the detection of known SNPs can be classified in two major groups i) platforms discriminating alternative alleles (mutant $v$ s.wild type) and ii) platforms detecting mutant alleles in a given DNA. The only platform able to detect and discriminate the alleles is MALDI TOF. There are also other allele discrimination methods, although not as powerful ad MALDI-TOF, including: i) PCRbased without fluorescent probe (ASA, SSCP and RFLP); ii) methods combining PCR with hybridization probes (FRET-based platforms, Invader assay and LNA Probes); iii) PCR-based with intercalating fluorescent dye as High Resolution Melting (HRM); iv) PCR required only for sample pre-treatment as Denaturing-High Performance Liquid Chromatography (D-HPLC).

The principal methods used to detect mutant alleles in given DNA, are: i) Pyrosequencing (require a PCR pre-treatment); ii) PNA mediated clamping-PCR; iii) Gene chip technologies; and iv) Sequencing methods.

New high-throughput sequencing technologies are incorporated in new platforms such as: $1 \mathrm{G}$ Genetic analyzer by Illumina/Solexa (Cambridge, UK), SOLiD by Life technologies (Foster City, CA, USA) and Genome Sequencer FLX by Roche Diagnostics (Brandfort CT USA). These "Next Generation DNA Sequencers" provide a consensus base accuracy of $99.99 \%$.

Non-PCR-based technologies, such as the Golden Gate Assay and DNA chip-based microarray are the latest development in the genotyping arena. These technologies are able to directly genotype genomic DNA without PCR amplification. These new technologies are not widely used in the general clinical laboratory setting compared to PCR-based methods.

The qualitative assessments of the polymorphic traits in genes are performed, without a necessary gold standard for the daily diagnostic routine (Table 1).

\section{Genotyping Costs}

Furthermore, trials evaluating the pharmacoeconomic impact of genotyping testing before therapy will likely provide answers for policy making in the merging of PGx testing into clinical practice. The primary aim of a cost-effectiveness analysis is to provide sufficiently robust information for decision-makers to allocate resources to healthcare interventions. Overviews of cost-effectiveness studies on PGx technologies are now available [5]. A relevant example is the NICE: a Diagnostic Advisory committee, which is willing to stimulate Pharma and Academic communities to produce a robust set of data, including design and data source in economic models of healthcare [6].

Only few studies have addressed the cost-effectiveness of pharmacogenomics testing implication in clinical practice. For example, Aquilante et al. [7] performed a study to compare the accuracy, the rapidity, and the cost of two methodologies used for genotyping a single variant in the cytochrome P450 (CYP) 2C9metabolizing enzyme gene: the cost/sample for 1 SNP detection was (US dollars) $\$ 1.90$ by PCRPyrosequencing and $\$ 3.14$ by RFLP. Other Authors, included thiopurine S-methyltransferase (TPMT) genotyping prior to 6-mercaptopurine treatment in paediatric Acute Lymphoblastic Leukaemia (ALL); the mean calculated cost from 4 European countries was $€ 2100,00$ per life-year considering low myelosuppression-related hospitalization; the cost for genotyping of TMPT mutation averaged around $€ 150,00$ [8]. In addition, Gold et al. [9], concluded that pharmacogenetic testing for UGT1A1*28 variant homozygosity before Ironotecan treatment may be cost effective. The genotype testing cost is averaged $\$ 103.00 \mathrm{z}$.

Early outline of genotyping cost for "home brew" pharmacogenomic tests is averaged about $€ 20,00$ per SNP.

\section{Conclusion and Future Outlook}

It is well known that PG tests performed before drug treatment, lower overall medical costs and provide higher quality of life and longer life expectancy. However, we still need a precise demonstration that PG tests offer an added value, in terms of relative cost and benefit.

The usefulness of the variants in clinical practice depends on improving the diagnostic prediction or fostering changes in prevention

\begin{tabular}{|c|c|c|c|}
\hline Genotyping methods to detect known SNP & Instrument mean costs $\S$ & Approximate reagent costs per SNP\$ & Approxim time-labour per SNP\# \\
\hline D-HPLC & ++++ & moderate & very fast \\
\hline SSCP & + & Low & very laborious \\
\hline Allele Specific Amplification (ASA) & + & very low & moderate \\
\hline $\begin{array}{l}\text { Restriction Fragment Length Polymorphism } \\
\text { (RFLP) }\end{array}$ & + & very low & very laborious \\
\hline $\begin{array}{l}\text { FRET probe Allelic Discrimination (Hyb Probe } \AA \\
\text { TaqMan } ₫ \text {, Beacons } ₫ \text { Scorpions } ₫ \text { ) }\end{array}$ & ++ & moderate & moderate \\
\hline Locked Nucleic Acid (LNA) probe & ++ & moderate & moderate \\
\hline Oligo ligation assay (SNPlex®) & +++ & high & Fast \\
\hline PCR-Invader $®$ Assay & +++ & high & Fast \\
\hline High resolution melting (HRM) & ++ & low & moderate \\
\hline Pyrosequencing* & +++ & high & Fast \\
\hline Peptide nucleic acid-mediated Clamping PCR* & + & moderate & moderate \\
\hline Gene Chip technology (LabOnChip) $^{*}$ & $+++/++++$ & very high & moderate \\
\hline Maldi-TOF & ++++ & very high & moderate \\
\hline Conventional sequencing* & ++ & low & moderate \\
\hline
\end{tabular}

$\S$ Approximate instrumentation list price were scored as $+(<10000 €) ;++(<50000 €) ;+++(<100000 €),++++(>100000 €)$.

\$Reagent costs were scored as very low $(<5 €)$, low $(<10 €)$, moderate $(<30 €)$, high $(<50 €)$, very high $(>50 €)$.

"Time-labour refers input needed to perform a single test of multiple samples. It were scored as very fast $(<1$ hour $)$, fast $(<4$ hours), moderate $(<1$ day), laborious $(<2$ day) very laborious ( $>2$ working day).

*No address to allelic discrimination (mutant vs. wild type).

Table 1: Most common platforms used for genotyping at molecular level. 
Citation: Monaco AD, Berretta M, Pugliese S, Valente D, Francia RD (2014) Early Outline Evaluation of Genotyping Costs of Pharmacogenomics. J Pharmacogenomics Pharmacoproteomics 5: 123. doi:10.4172/2153-0645.1000123

Page 3 of 3

or treatment strategies. There is a compelling need for detailed and extensive studies to establish the cost and effectiveness of genotyping. Although there are still unanswered questions, PG researchers have generated and improved specific tools for novel treatment and handling of cancer patients. With new PG markers being identified and validated, physicians will have ways and means to tailor specific drugs to individual genetic profiles.

Over the next few years, the emergence of molecular resistance to new therapies as a result of genomic alterations in cancer will drive diagnostics companies to develop new tests for individualized therapy. The future implementation of the methods for genotyping will lead to personalized treatment and eventually will shift the balance from disease relapse toward disease eradication. Therefore, it is essential that pharmaceutical and biotechnology companies join the efforts to develop accurate and cheap tests for routine diagnostics in pharmacogenomics.

\section{Authors' Conflict of Interest Disclosure}

The authors stated that there are no conflicts of interest regarding the publication of this article.

\section{References}

1. Deverka PA (2009) Pharmacogenomics, evidence, and the role of payers. Public Health Genomics 12: 149-157.
2. Di Francia R, Berretta M, Catapano O, Canzoniero LM, Formisano L (2011) Molecular diagnostics for pharmacogenomic testing of fluoropyrimidine basedtherapy: costs, methods and applications. ClinChem Lab Med 49: 1105-1111.

3. Crowford JM, Aspinall MG (2012)The business value and cost-effectiveness of genome medicine. Personalized Medicine 9: 265-286.

4. Balboa E, Duran G, Lamas MJ, Gomez-Caamaño A, Celeiro-Muñoz C, et al (2010) Pharmacogenetic analysis in neoadjuvantchemoradiation for recta cancer: high incidence of somatic mutations and their relation with response. Pharmacogenomics 11: 747-761.

5. Dhalla IA, Garner S, Chalkidou K, Littlejohns P (2009) Perspectives on the National Institute for Health and Clinical Excellence's recommendations to use health technologies only in research. Int $\mathrm{J}$ Technol Assess Health Care 25 : 272-280.

6. Payne K, Shabaruddin FH (2010) Cost-effectiveness analysis in pharmacogenomics. Pharmacogenomics 11: 643-646.

7. Aquilante CL, Lobmeyer MT, Langaee TY, Johnson JA (2004) Comparison of cytochrome P450 2C9 genotyping methods and implications for the clinical laboratory. Pharmacotherapy 24: 720-726.

8. van den Akker-van Marle ME, Gurwitz D, Detmar SB, Enzing CM, Hopkins MM et al. (2006) Cost-effectiveness of pharmacogenomics in clinical practice: a case study of thiopurine methyltransferase genotyping in acute lymphoblastic leukemia in Europe. Pharmacogenomics 7: 783-792.

9. Gold HT, Hall MJ, Blinder V, Schackman BR (2009) Cost effectiveness of pharmacogenetic testing for uridine diphosphate glucuronosyl transferase $1 \mathrm{~A} 1$ before irinotecan administration for metastatic colorectal cancer. Cancer 115 3858-3867. 